DOI: https://doi.org/10.46296/yc.v5i8edespjun.0096

\title{
EVOLUCIÓN DE LA NORMA ISO 14001 Y SU IMPLEMENTACIÓN EN EL ECUADOR
}

\section{EVOLUTION OF THE ISO 14001 STANDARD AND ITS IMPLEMENTATION IN ECUADOR}

\author{
Zambrano-Carranza Doris Maribel ${ }^{1 *}$; Pérez-Parra Jean Carlos ${ }^{2}$; Perero-Espinoza \\ Galo Arturo ${ }^{3}$ \\ ${ }^{1}$ Universidad Técnica de Manabí, UTM. Portoviejo, Ecuador. \\ ${ }^{2}$ Dpto. de Química. Instituto de Ciencias Básicas. Universidad Técnica de Manabí, \\ UTM. Portoviejo, Ecuador. \\ ${ }^{3}$ Carrera de Ingeniería Industrial. Facultad de Ciencias Matemáticas, Físicas y \\ Químicas. Universidad Técnica de Manabí, UTM. Portoviejo, Ecuador.
}

*Correo: dzambrano9587@utm.edu.ec

\begin{abstract}
Resumen
La norma ISO 14001, es un marco que define las reglas para integrar las preocupaciones ambientales en las actividades de las organizaciones con el fin de controlar los impactos ambientales y así conciliar los requisitos operativos de la organización con el respeto al ambiente. Partiendo de ello el objetivo del presente artículo es analizar como la norma ISO 14001 ha evolucionado a nivel internacional y como ha sido implementada en Ecuador. Proceso que se llevó a cabo mediante un análisis teórico que permitió establecer que: los numerosos beneficios que ofrece la norma, lo que genera un cambio positivo para los diferentes sectores económico, permitiendo un enfoque integral y activo de los aspectos ambientales de las organizaciones. Respecto a cómo la generación de certificaciones a nivel continental es posible establecer que en Africa presentó un 39,53\% de creciente de certificaciones, seguido de Asia con el $29,91 \%$, América con $3,99 \%$ y Europa con un crecimiento de 1,28\%, estableciendo así la intención internacional de la aplicación de la norma ISO 14001. En países como Ecuador, Colombia, Perú y Uruguay, los sectores de mayor aplicación son el sector transporte, almacenamiento y comunicación, el sector construcción y otros sectores no catalogados. En el caso ecuatoriano, es necesario que se aumente el número de certificaciones siendo que el mismo se encuentra en el sexto puesto en comparación con los países del continente americano.
\end{abstract}

Palabras claves: Sistema de gestión ambiental, Ventajas de la norma ISO 14001.

\begin{abstract}
The ISO 14001 standard is a framework that defines the rules to integrate environmental concerns into the activities of organizations in order to control environmental impacts and thus reconcile the operational requirements of the organization with respect for the environment. Based on this, the objective of this article is to analyze how the ISO 14001 standard has evolved internationally and how it has been implemented in Ecuador. Process that was carried out through a theoretical analysis that allowed to establish that: the numerous benefits offered by the standard, which generates a positive change for the different economic sectors, allowing a comprehensive and active approach to the environmental aspects of organizations. Regarding how the generation of certifications at a continental level, it is possible to establish that in Africa there was a $39.53 \%$ growth in certifications, followed by Asia with $29.91 \%$, America with $3.99 \%$ and Europe with a growth of $1,28 \%$, thus establishing the international intention of applying the ISO 14001 standard. In countries such as Ecuador, Colombia, Peru and Uruguay, the sectors with the greatest application are the transport, storage and communication sector, the construction sector and
\end{abstract}

Información del manuscrito:

Fecha de recepción: 02 de abril de 2021.

Fecha de aceptación: 31 de mayo de 2021.

Fecha de publicación: 01 de junio de 2021. 
other non-sectors. cataloged. In the Ecuadorian case, it is necessary to increase the number of certifications since it is in sixth place compared to the countries of the American continent.

Keywords: Environmental management system, Advantages of the standard ISO 14001.

\section{Introducción}

El incremento de la explotación y transformación de recursos naturales no sostenibles, así como el agotamiento de los mismos, acompañados con la constante liberación de contaminantes peligrosos han desencadenado un evidente deterioro del ambiente a través del tiempo (Bazán y Bruno,(2016).

Coello (2018), consideró que, de forma paralela al cambio ambiental se produce también un cambio social global. Esto principalmente se debe a la propia dinámica interna del sistema mundial, que se caracteriza por la constante expansión demográfica, los procesos de desarrollo económico y la tendencia hacia la globalización de la economía y de la tecnología, por medio de potentes redes de interdependencia.

Lara (2014), estableció que, en países de mayor crecimiento suyos procesos productivos se basan en la industria como es el caso de Estados Unidos, Japón y Rusia emplean más del $80 \%$ de los recursos naturales del planeta, a pesar de que los mismos solo cuentan con la cuarta parte de la población mundial; esto se presenta esencialmente por las exigencias propias del desarrollo tecnológico que se lleva a cabo en estos países, generando además una afectación ambiental equivalente al desgaste de los recursos.

Esta afectación ambiental ha traído consigo un proceso de concienciación en el colectivo social, así como movimientos ecológicos centrados en frenar los efectos nocivos que se producen de los procesos de producción y de consumo de mercancías en el agotamiento de los recursos, en la desestructuración de los ecosistemas y en la desintegración cultural de los pueblos (Guzmán Ramos, 2001, pág. 4). 
Castaño y Román (2016) consideraron que, en este contexto, la norma ISO 14000 de Sistemas de Gestión Ambiental nace como un compromiso del sustento del ambiente con un enfoque que pudiera ser aplicado globalmente. En octubre de 1996, el lanzamiento del primer componente de la serie de estándares ISO 14000 salió a la luz, a revolucionar los campos empresariales, legales y técnicos.

Ecuador en los últimos años, desde el sector gubernamental ha evidenciado varias iniciativas para promover el cuidado y manejo del ambiente, entre las que se pueden mencionar instrumentos legales como la Constitución de la República del Ecuador (2008), en sus artículos $14,15,66,71,278,395,403$ y 408 , en los que se establecen lineamientos de protección ambiental. También se consideran aspectos ambientales en la Ley de Gestión Ambiental (2004) y el Código Orgánico Ambiental (2017).

Estos instrumentos normativos legales a nivel nacional han generado que las instituciones tanto públicas como privadas centren sus esfuerzos en fortalecer de manera oportuna sus sistemas de gestión ambiental, es así como han emprendido el camino para la obtención de la certificación ISO 14001:2015.

Partiendo de lo previamente mencionado el presente artículo tiene como finalidad el análisis de como la norma ISO 14001 ha evolucionado a nivel internacional y como ha sido implementada en Ecuador.

\section{Desarrollo}

\section{Norma ISO 14001:2015}

La Organización Internacional de Normalizaciones (ISO), que fue fundada en 1946, se conforma por delegaciones de carácter gubernamentales, así como no gubernamentales, y en la actualidad representan a más de 100 agrupaciones o países miembros subdivididos en una serie de subcomités; esta se encarga del desarrollo de guías que contribuirán al mejoramiento ambiental. Las mismas no se encuentran afiliadas a las naciones unidas, ni a ninguna organización europea (Ccoscco Vasquez, 2017, pág. 93). 
A pesar de que las normas que desarrolla la ISO son diseñadas para el sector privado y su aplicación es de carácter específicamente voluntario, es común que muchos organismos del tipo gubernamental ha decidid convertir una norma ISO en una disposición obligatoria o legal (Rojas González, 2010).

La norma ISO 14000 pertenece a un grupo de normas internacionales cuya aplicación es de carácter voluntario cuya finalidad se centra en los sistemas de gestión ambiental; de estas familias de normas, la primera em generarse fueron las ISO 14001 publicada en 1996 direccionada en sistemas de gestión ambiental, contando con especificaciones y directrices para su utilización. Posteriormente y tras un largo procesos de revisión, en el 15 de septiembre de 2015 se publicó la ISO 14001:2015 misma que a la actualidad se encuentra vigente. Esta norma no prescribe requisitos de actuación, salvo el de compromiso de mejora continua y la obligación de cumplir con la legislación aplicable (Cubas López \& Mendoza Cabrera, 2018, pág. 35)

La ISO 14001 es una norma que proporciona los lineamientos para la implantación de un SGA, orientando a las organizaciones pequeñas, medianas y grandes, al manejo y control de los impactos que producen sus actividades en el medio ambiente, reducirlos o incluso eliminarlos (Albornoz Aldeán, 2017, pág. 29).

La norma ISO 14001 "constituye un enfoque sistemático sobre los aspectos ambientales de una organización y es una herramienta que posibilita a una organización, cualquiera que sea su tipo o tamaño, controle el impacto de sus actividades, productos o servicios sobre el medio ambiente". (Norma ISO 14001, 2015, pág. 17)

El estándar hace que las empresas sean mucho más sostenibles, desarrollen tecnologías limpias, se especialicen en el manejo de todos los aspectos ambientales y cumplan con la legislación ambiental vigente (Albornoz Aldeán, 2017, pág. 187).

El objetivo de la norma del sistema de gestión ISO 14001 es especificar requisitos y directrices generales que, cuando se sigan, proporcionen una seguridad razonable de que los resultados del sistema 


tendrán un $\begin{array}{r}\text { impacto } \\ \text { medioambiental }\end{array}$
mínimo y un rendivo
medioambiental mejorado (Del
Castillo Palacios, 2018, pág.
154)

Enrriquez (2009), establece que el propósito fundamental de los sistemas de gestión ambiental basados en la norma ISO 14001 es:

- Identificar aspectos ambientales significativos, al igual que la legislación aplicable y otros requisitos.

- Formular y asumir un compromiso enmarcado en la política ambiental institucional.

- Lograr los objetivos y metas previstas, mediante las cuales se previene la contaminación, se cumple con los requisitos y se mejora el desempeño ambiental y la toma de conciencia respecto al ambiente.

\section{Ventajas de la norma ISO 14001:2015}

Las ventajas de utilizar un sistema de gestión ambiental se encuentran orientadas a garantizar un enfoque holístico de los impactos ambientales, centrándose únicamente en aspectos y procesos críticos, haciendo uso de enfoques maduros probados a lo largo del tiempo y reconocidos en todo el mundo, estableciendo relaciones positivas con los entes reguladores nacionales y extranjeros (Espín Guerrero, 2018, pág. 83)

Alzate et al (2018), señalaron que la ISO 14001 actualmente es considerada como uno de los estándares que actualmente presentan un mayor número de certificaciones a nivel mundial, lo que la convierte en un imperativo para muchas empresas, esto esencialmente por los potenciales beneficios que trae consigo, debido a que la misma facilita una reducción del impacto ambiental que se genera por las actividades económicas; El desarrollo del SGA, a partir de la ISO 14001 disminuye la generación de externalidades negativas, proporciona un marco para mejorar la ecoeficiencia, favorece la imagen corporativa, posibilita la disminución de costos y aumento en los ingresos.

Coello (2018), planteó que la adopción de un SGA puede ayudar a una organización a: 
- Cumplir con las leyes y regulaciones ambientales.

- Mejorar su prestigio y reputación con el personal, las organizaciones asociadas y otras partes interesadas.

$\begin{array}{llr}\text { - Adaptarse a un entorno } & \text { un } \\ \text { cambiante (ya sea } & \text { sus } \\ \text { operaciones } & \text { o } & \text { sus } \\ \text { productos/servicios). } & & \end{array}$

Bases metodológicas para la aplicación de la norma ISO 14001:2015

La Norma ISO 14001 se desarrolla en torno al modelo de mejora conocido como el Ciclo de Deming: Planificar, Hacer, Verificar y Actuar (PHVA), un proceso iterativo que debe aplicarse regularmente para garantizar que los beneficios se cumplan y se mantenga el estándar (Ayoví (2018).

El modelo PHVA proporciona un proceso repetitivo, usado por las organizaciones para lograr la mejora continua. Ayoví (2018) establece que, los componentes operativos principales de un Sistema de Gestión Ambiental basado en la norma ISO 14001:2015 se pueden agrupar tal como se presentan en la tabla 1.

Tabla 1. Componentes operativos de SGA-ISO 14001:2015

1. Planificar: Realizar una revisión medioambiental e identificar lo que afecta o afectará a la organización. Seguido de la definición de objetivos, metas y planes de acción para la mejora del desempeño medioambiental, lo cual debe estar alineado con la política ambiental establecida. Entre las subetapas se pueden mencionar:

- Análisis de los aspectos ambientales que se encuentran relacionados con el accionar de la institución.

- Establecer los requisitos legales que se deben considerar.

- Planificar las acciones que se van a llevar a cabo.

- Establecimiento de los objetivos ambientales, metas y programas.
2. Hacer: Implementar planes de acción de gestión ambiental, los cuales pueden estar conformados por:

- Recursos, responsabilidades y autoridad.

- Competencia, entrenamiento y conciencia.

- Comunicación interna y externa.

- Información documentada.

- Creación y actualización.

- Control de información documentada.

- Planificación y control operacional.

- Preparación y respuesta ante emergencias. 
- Planificación de acciones para lograr los objetivos ambientales.

3. Verificar: La ISO 14001:2015 establece procesos de seguimiento y medición de la política ambiental, incluyendo los compromisos, los objetivos ambientales y los criterios de operación, como:

- Evaluación del desempeño.

- Seguimiento, medición, análisis y evaluación.

- Evaluación del cumplimiento.

- Auditorías internas.

- Programa de auditoría interna.

- Revisión por la dirección.
4. Actuar: Adoptar medidas para la mejora del desempeño ambiental de manera periódica, entre las que se pueden mencionar:

- Acciones correctivas.

- Aplicación de procesos de mejora continua.

- Revisión de gestión del SGA.

Fuente: Escuela Europea de Excelencia (2015).

A grandes rasgos es posible considerar que la norma ISO 14001:2015, es un marco que define las reglas para integrar las preocupaciones ambientales en las actividades de la organización con el fin de controlar los impactos ambientales y así conciliar los requisitos operativos de la organización con el respeto al medio ambiente.

\section{Principales desafíos que afrontan} las instituciones públicas en la

\section{transición a la norma ISO 14001:2015}

Enrriquez (2009), señaló que, entre los desafíos que afrontan las instituciones en la aplicación de la norma ISO 14001:2015, independientemente si la empresa es de origen público o privado, es posible mencionar los siguientes:

Contexto de la organización: La ISO 14001 exige que sean identificados y comprendidos los factores que pueden perjudicar el alcance de los objetivos. La norma también amplía el concepto de partes interesadas, más allá de clientes, socios y accionistas.

\section{Pensamiento basado en riesgos:}

Las organizaciones precisan considerar los riesgos durante la toma de decisiones. Ya sea a nivel estratégico, táctico u operacional. En algunos segmentos eso es algo natural, en otros la complejidad puede ser mayor. 
Abordaje de procesos: Definir los procesos relevantes para el negocio y atribuir indicadores que comprueben su eficacia. Ese desafío está presente desde las versiones antiguas de la norma, y sigue siendo relevante en la versión 2015.

\section{Liderazgo y compromiso:}

Demostrar el compromiso con los sistemas de gestión ambiental, y garantizar la integración de esos requisitos con los procesos de negocios, requiere una dedicación mayor de los ejecutivos en el proceso de certificación.

\section{Distribución geográfica: En} organizaciones con varias unidades de negocio, garantizar que la transición sea consistente eleva la complejidad del proceso. La tecnología puede ser una gran aliada en la diseminación del conocimiento.

Tiempo: La planificación anticipada, la evaluación de los vacíos, la concienciación, educación y el entrenamiento, pueden disminuir la exposición a los riesgos durante el proceso transitorio.

Terminología: La terminología evolucionó con la norma. El proceso de transición exigirá gran esfuerzo para concienciar e implementar estas novedades en todos los niveles y procesos.

Productividad y Resultados: La ISO 14001:2015 trae un enfoque mayor en aspectos como "productividad" y "desempeño". Con los requisitos básicos tratando las cuestiones de compromiso de los liderazgos, pensamiento basado en el riesgo y expectativas de las partes interesadas, las organizaciones pueden concentrarse en alcanzar los resultados esperados.

\section{Aplicación de la norma ISO 14001} en el panorama mundial.

Desde la publicación de la primera versión de la norma ISO 14001 de SGA, ha sido creciente la adopción de este estándar internacional en las organizaciones que buscan dar respuesta a la demanda de un entorno cada vez más dinámico y exigente (Salazar Terán, 2017, pág. 65).

La decisión de adoptar un SGA basado en la ISO 14001 se ha asociado al grado de desarrollo de los países, al tamaño de la empresa $y$ al nivel organizacional (Huatuco Mamani, 2019). 
Alzate et al (2018), señalaron que a partir de los estudios desarrollados sobre la difusión de la ISO 14001 a nivel mundial, se ha evidenciado que la distribución del número de certificaciones por continentes e inclusive por países es desigual.

Los primeros continentes en adoptar la norma ISO 14001, a mediados de la década de los 2000, fueron América del Norte y Europa Occidental. Posteriormente, y a medida que aumentaron las relaciones comerciales, se elevó el número de certificaciones en el este de Asia, extendiéndose subsecuentemente a América del Sur y Euro-Asia.

En la figura 1 se muestra el número de certificaciones ISO 14001 a nivel mundial desde el año 2007 hasta el 2016, y en esta se puede apreciar un aumento en cuanto al número de certificaciones de ISO 14001, pasando de 154572 certificados ISO 14001 en el 2007 a 346189 certificados en 2016, en todo el mundo, incrementándose en más del doble el número de certificaciones en 10 años. Con un crecimiento anual de certificaciones para el año 2016 del $17 \%$ con respecto al 2015.

Figura 1. Número de certificaciones ISO 14001 en el mundo desde 2007 hasta 2016.

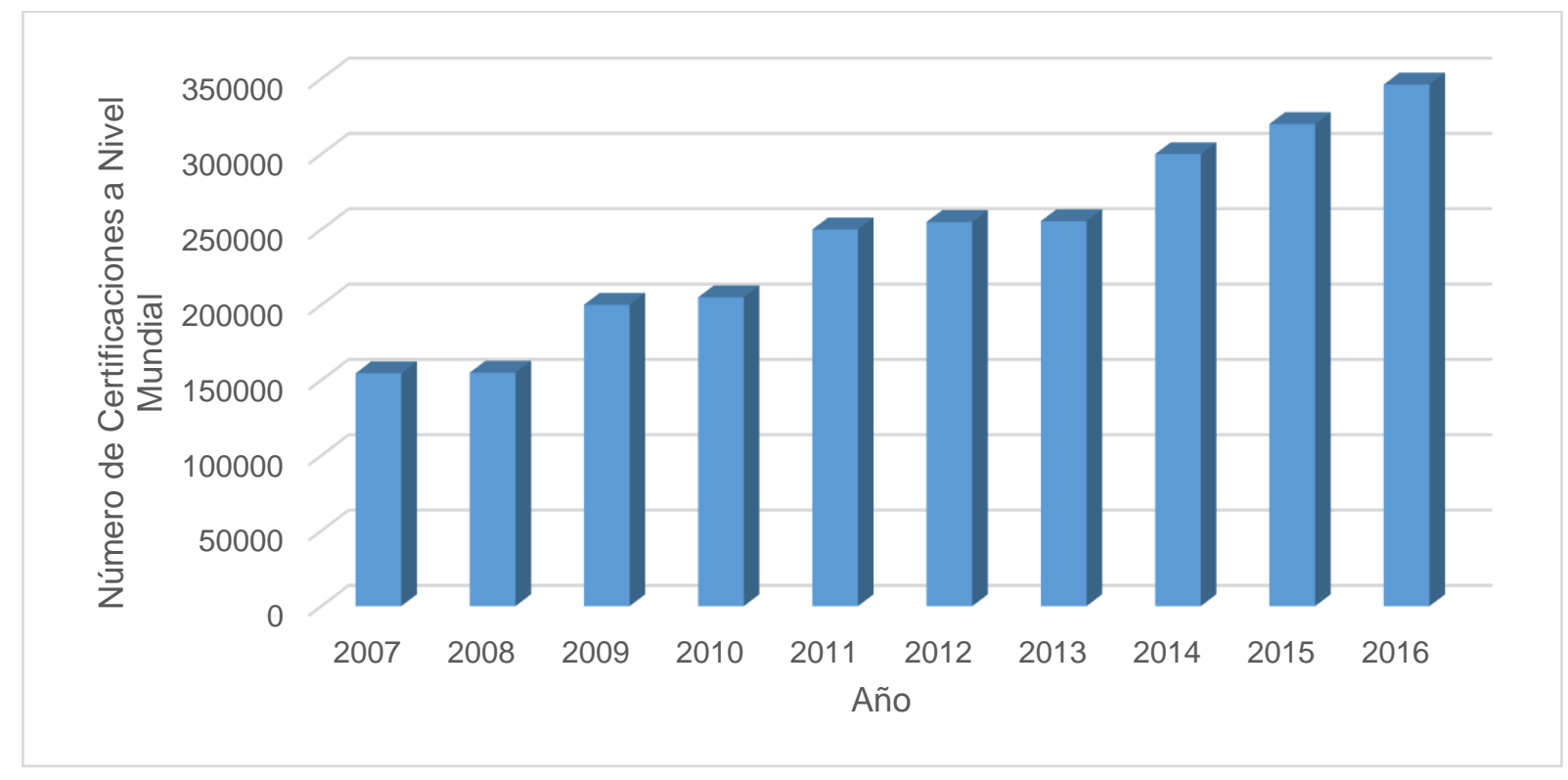

Fuente: Informe ISOsurvey (International Organization for Standardization, 2016).

Según el informe International Organization for Standardization
(2016) los países que presentaron mayor crecimiento en el número de 
certificaciones en ISO 14001 emitidas para el año 2016, fueron China, Japón, Italia, Reino Unido, España, Rumania y Alemania. Estas estadísticas evidencian la importancia que ha cobrado la certificación en países desarrollados, y el relego de los países con economías emergentes.

A diferencia de los países mencionados, el caso de los países de América del Centro y Sur sigue siendo muy bajo en comparación con el número de certificaciones a nivel mundial; Según el Informe ISOsurvey (2016) para el año 2016 se reportan un total de 10486 certificaciones, lo que equivale al $3 \%$ del número de certificaciones en el mundo. El panorama durante los últimos tres años es desalentador, con porcentajes de crecimiento del $2 \%$ para el 2014 y 2015 , y del $4 \%$ en el 2016. Este escenario probablemente se encuentre asociado al contexto cultural, socioeconómico y político de los países, que afectan la toma de decisiones frente a la adopción de herramientas de gestión como la ISO 14001, y en segunda instancia al periodo de transición en el que se encuentra el referente normativo.

\section{Aplicación de la norma ISO 14001 por continente.}

Según el informe ISOsurvey (2016), África pasó de tener 2545 certificaciones en el año 2014, a 3024 en 2015 y finalmente a 3551 en 2016. En América en el 2014 se llegó a la cifra de 10084, en 2015 se elevó a 10097 y finalmente en 2016 tenían 10486 certificados en ISO 14001.

En Europa el número de certificados en el año 2014 fue de 119072 certificados, para el 2015 se incrementaron a 119754 certificados y en el año 2016 se llegó a un total de 120595 certificados en ISO 14001. En Asia es donde mayor número de certificados es posible encontrar, en 2014 con 145877 certificados ISO 14001, en 2015 aumento a 165616 certificados y en 2016 contaban con 189505 certificados ISO 14001. Estos datos se encuentran detallados en la figura 2. 
Figura 2. Número de certificaciones ISO 14001 por continente en los años 2014, 2015 y 2016.

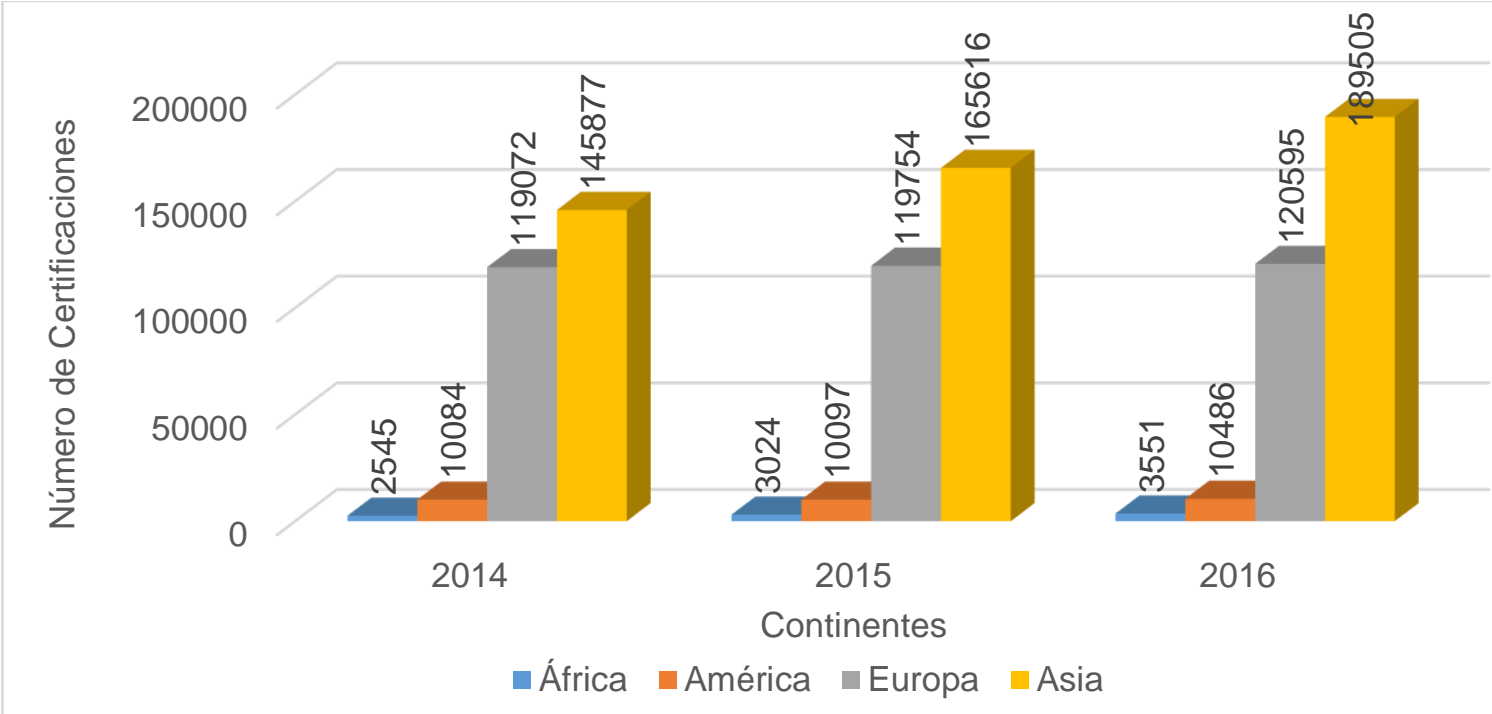

Fuente: Informe ISOsurvey (International Organization for Standardization, 2016).

La figura 3 muestra una comparación de las variaciones anuales considerando la tasa de crecimiento porcentual del incremento de las certificaciones ISO 14001 durante los periodos 2014, 2015 y 2016.

Figura 3. Tasa de crecimiento de la emisión de certificaciones ISO 14001 por continente en los periodos del 2014 al 1016.

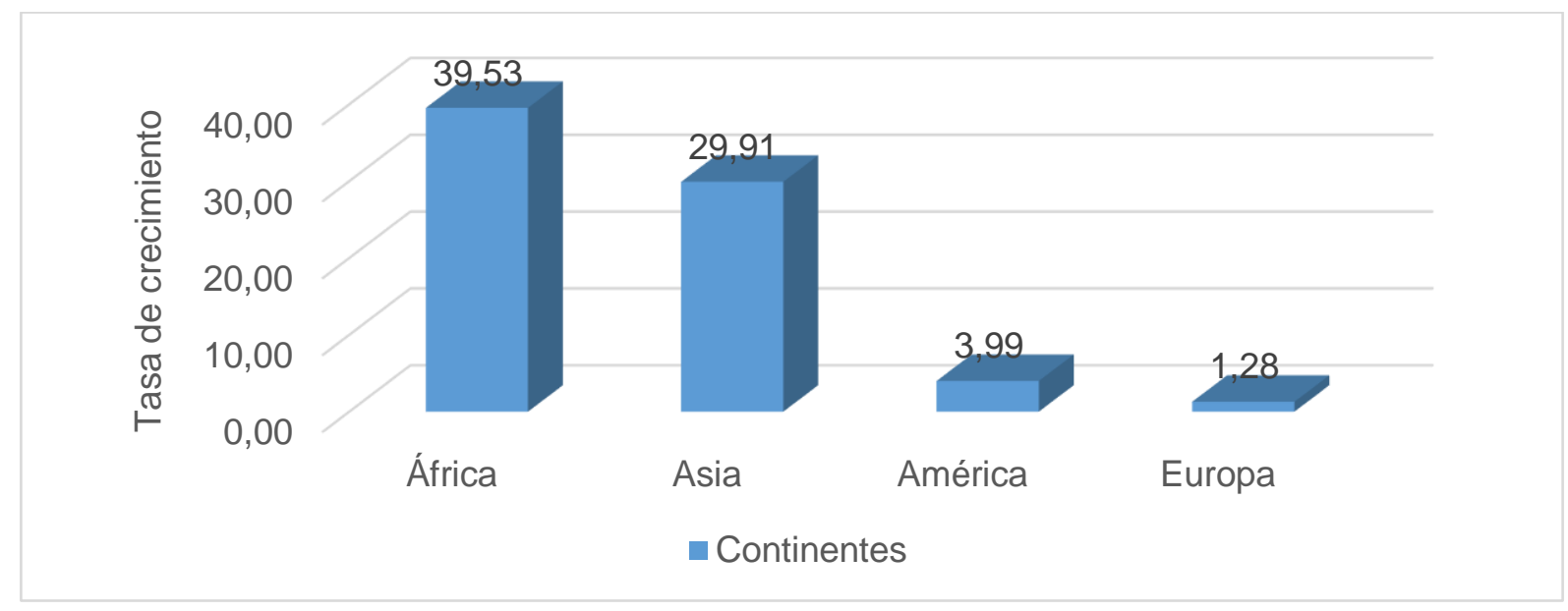

Fuente: Informe ISOsurvey (International Organization for Standardization, 2016).

De los datos de la figura 3 se puede apreciar que África presenta una mayor tasa de crecimiento en cuanto a emisión de las certificaciones, siendo esta del $39,53 \%$, seguido de
Asia con el $29,91 \%$, América con $3,99 \%$ y Europa con un crecimiento de $1,28 \%$. 
Aplicación de la norma ISO 14001:2015 en América del sur para el 2019.

En lo que refiere al panorama de América del Sur, específicamente para el año 2019, el país con mayor número de certificaciones es Colombia con un total de 3341 es decir el 31,45\%, seguido de Brasil con 3166 certificaciones $(29,80 \%)$, en tercer lugar, se ubica Argentina con 1908 certificaciones $(17,96 \%)$. El caso ecuatoriano para el mismo periodo presentó 200 certificaciones, es decir el 1,88\% del número de certificaciones en América del Sur, estando posicionado en el sexto lugar en relación con los países de la región. Estos datos se representan en la figura 4.

Figura 4. Certificaciones ISO 14001:2015 en América del sur para el 2019.

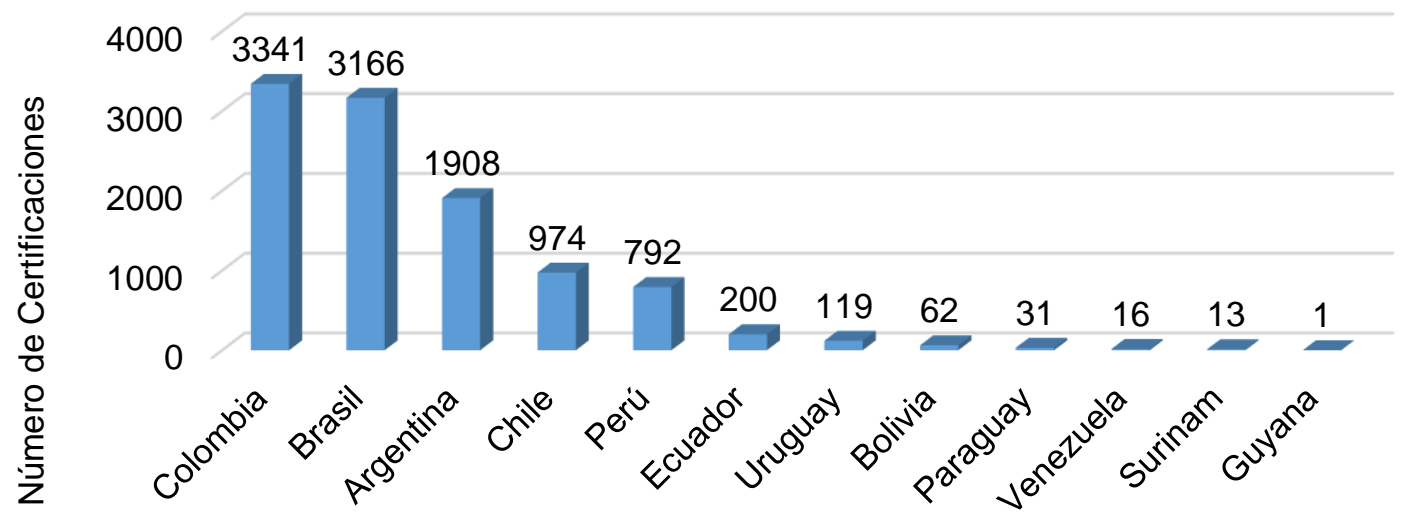

Países de América del Sur

Fuente informe ISOsurvey (International Organization for Standardization, 2019)

Dinámica de las certificaciones ISO 14001:2015 en Ecuador, Colombia y Perú durante el periodo del 2019.

En lo que refiere al comportamiento de las normas ISO 14001:2015 en Ecuador, Colombia, Perú y Uruguay, el informe de la Organization for Standardization (2019), detalla que para el año 2019 se generaron 3341 certificaciones en Colombia, 792 en Perú y 200 en Ecuador, como se lo muestra en la figura 5 . 
Figura 5. Certificaciones ISO 14001:2015 en Colombia, Perú, Ecuador y Uruguay durante el periodo del 2019.
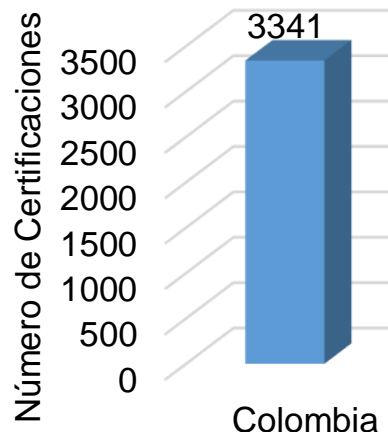

Colombia

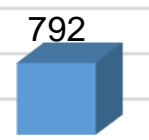

Perú

Países

200

Ecuador
119

Uruguay

Fuente: Informe ISOsurvey(International Organization for Standardization, 2019)

En este sentido en Colombia, para el año 2019 se generaron 3341 , siendo el sector construcción y el de transporte, almacenamiento y comunicación los que presentaron un mayor número de certificaciones, 558 y 353 respectivamente.

En lo que respecta al número de certificaciones en Perú, para el año 2019 se generaron 792, estando la mayor parte de certificaciones en el sector construcción 107 y 78 en el sector de transporte, almacenamiento y comunicación.

En lo que respecta al número de certificaciones en Ecuador en el año 2019 se generaron 200 certificaciones en los diferentes sectores económicos, estando la mayor parte entre tres sectores específicos; 25 de ellas se presentaron en el sector comercio mayorista y minorista, reparación de vehículos de motor, motocicletas y artículos personales y domésticos; 22 en el sector transporte, almacenamiento y comunicación, y 16 certificaciones en el sector de minas y canteras.

En el caso de Uruguay en el año 2019 se expidieron 119 certificaciones, estas se presentaron mayormente concentradas en el sector de la construcción con 29 certificaciones, y el sector transporte, almacenamiento y comunicación con 17 certificaciones.

Para establecer una mejor comparación en función al tamaño del país se estableció un índice que 
relaciona el número de certificaciones por cada cien mil en la figura 6 .

Figura 6. Certificaciones por cada cien mil habitantes ISO 14001:2015 en Colombia, Perú, Ecuador y Uruguay durante el periodo del 2019.
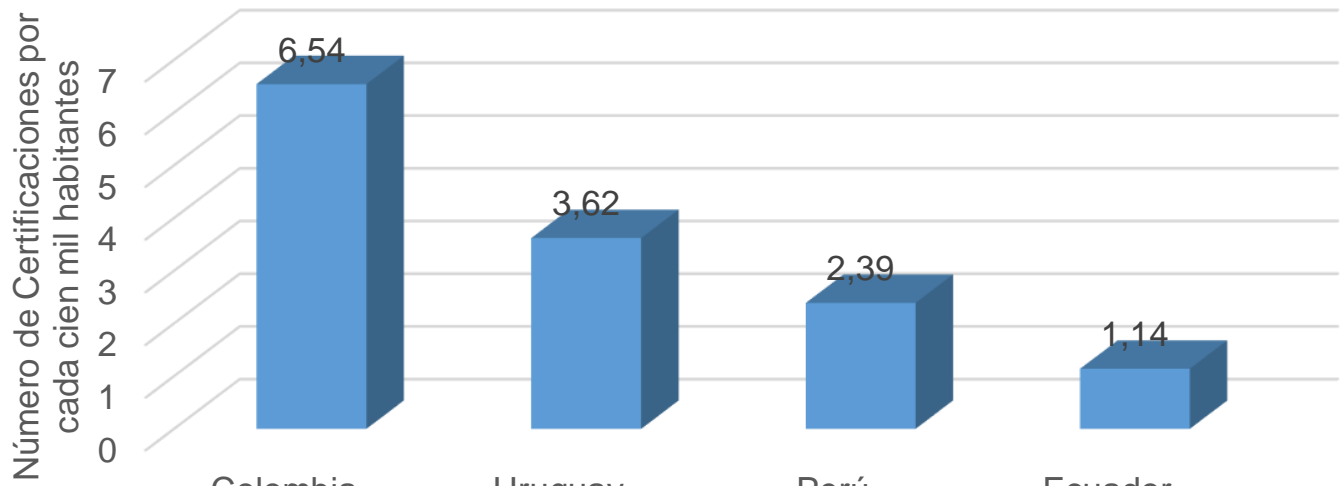

Colombia

Uruguay

Perú

Ecuador

Países

Fuente Informe ISOsurvey(International Organization for Standardization, 2019)

Según el índice establecido para el proceso de comparación Colombia presentó 6,54 certificaciones por cada cien mil habitantes, seguido de Uruguay con 3,62 certificaciones, Perú genero 2,39 certificaciones y Ecuador genero unas 1,14 certificaciones por cada cien mil habitantes.

\section{Conclusiones}

En cuanto a la información teórica, los resultados indican que la Norma ISO 14001 ofrece numerosos beneficios, lo que genera un cambio positivo para los diferentes sectores económicos, permitiendo un enfoque integral y activo de los aspectos ambientales de las organizaciones, además de mejorar la competitividad a nivel nacional e internacional.

Respecto a cómo la generación de certificaciones basadas en la Norma ISO 14001:2015 a nivel continental es posible establecer un crecimiento sostenido de la misma, siendo que África presento un $39,53 \%$ de creciente de certificaciones, seguido de Asia con el 29,91\%, América con $3,99 \%$ y Europa con un crecimiento de 1,28\%, estableciendo así la intención internacional de la aplicación de la norma ISO 14001:2015. 
Respecto a cómo se han desarrollado las certificaciones ISO 14001:2015 en países como Ecuador, Colombia, Perú y Uruguay, los sectores de mayor aplicación son el sector transporte, almacenamiento y comunicación, el sector construcción y otros sectores no catalogados. En el caso ecuatoriano, es necesario que se aumente el número de certificaciones a base de la Norma ISO 14001:2015 siendo que el mismo se encuentra en el sexto puesto en comparación con los países del continente americano.

\section{Bibliografía}

Albornoz Aldeán, M. K. (2017). Diseño de un sistema de gestión ambiental basado en ISO 14001:2015 para el departamento ambiental del gobierno autónomo descentralizado del cantón Pedro Vicente Maldonado, provincia de Pichincha, república el Ecuador. Quito: Universidad Técnica Particular de Loja. http://dspace.utpl.edu.ec/han dle/123456789/17982

Alzate lbáñez, A. M., Ramírez Ríos, J. F., \& Alzate Ibáñez, S. M. (2018). El modelo de gestión ambiental ISO 14001: evolución y aporte a la sostenibilidad organizacional. Revista Chilena de Economía y Sociedad. https://rches.utem.cl/articulos/ el-modelo-de-gestionambiental-iso-14001evolucion-y-aporte-a-lasostenibilidadorganizacional/\#: :text=La\%2 0ISO $\% 2014001 \% 20$ se $\% 20$ ha ,facilita\%20la\%20reducci\%C 3\%B3n\%20del\%20impacto

Alzate, A. M., Ibáñez, S. M., \& Ramírez Ríos, J. F. (2018). El modelo de gestión ambiental ISO 14001: evolución y aporte a la sostenibilidad organizacional. Revista Chilena de Economía y Sociedad.

https://rches.utem.cl/articulos/ el-modelo-de-gestionambiental-iso-14001evolucion-y-aporte-a-lasostenibilidadorganizacional/\#: :text=La\%2 0ISO $\% 2014001 \% 20$ se $\% 20$ ha ,facilita\%20la\%20reducci\%C 3\%B3n\%20del\%20impacto

Asamblea Constituyente. (2008). Constitución del Ecuador. Montecristi.

https://www.acnur.org/fileadm in/Documentos/BDL/2008/67 16.pdf

Asamblea Nacional. (2004). Ley de Gestión Ambiental. Registro Oficial Suplemento 418 de 10sep-2004.

https://www.ambiente.gob.ec/ wp- 
content/uploads/downloads/2 012/09/LEY-DE-GESTIONAMBIENTAL.pdf

Ayoví Barrio, A. J. (2018). Producción de abono orgánico a partir de la gallinaza, como sistema de gestión ambiental, ISO 14001. Guayaquil - Ecuador: Universidad de Guayaquil. http://repositorio.ug.edu.ec/bit stream/redug/29339/1/TESIS \%20AMELIA\%20AYOVI\%20I MPRIMIR\%2026-022018\%20Modificado.pdf

Bazán Díaz, A. O., \& Bruno Chávez, G. J. (2016). Propuesta de implementación de un sistema de gestión medioambiental según la norma ISO 14001:2015 en un laboratorio de productos farmacéuticos. Lima - Perú: Universidad Nacional Mayor de San Marcos. https://cybertesis.unmsm.edu .pe/bitstream/handle/20.500.1 2672/4893/Bazan_da.pdf?se quence $=1$ \& isAllowed $=y$

Castaño Ayala, M. A., \& Román Cárdnas, L. A. (2016). Diseño del sistema de gestión ambiental con base en la norma ISO 14001 para la organización privada: Seguridad Sara Ltda, ubicada en la ciudad de Bogotá Cundinamarca. Bogotá: Universidad de Ciencias Aplicadas y Ambientales (UDCA). https://repository.udca.edu.co /bitstream/11158/530/1/PRO YECTO\%20DE\%20GRADO \%202016\%20final.pdf

Ccoscco Vásquez, R. (2017). Implementación de Sistema de Gestión Ambiental según norma ISO 14001:2015 en la empresa de Transporte de personal REMISSE 21. Arequipa Perú: Universidad Nacional de San Agustín de Arequipa.

http://repositorio.unsa.edu.pe/ bitstream/handle/UNSA/5837/ AMccvar.pdf?sequence $=1$ \&is Allowed $=\mathrm{y}$

Coello Montiel, E. V. (2018). Diseño de un sistema de gestión ambiental para efectuar en la empresa AGRISON S.A, empleando la norma ISO 14001-2015. Guayaquil Ecuador: Universidad de Guayaquil.

http://repositorio.ug.edu.ec/bit stream/redug/35079/1/TESIS \%20FINAL\%20ERIKA\%20C OELLO\%2003.9.2018.pdf

Cubas López, G. F., \& Mendoza Cabrera, K. Y. (2018). Diseño de un sistema de gestión ambiental basado en la norma ISO 14001:2015, aplicado a la empresa Atlética S.R.L. Chiclayo: Universidad Católica Santo Toribio de Mogrovejo.

http://tesis.usat.edu.pe/bitstre $\mathrm{am} / 20.500 .12423 / 1464 / 1 / \mathrm{TL}$ 
CubasLopezGina_MendozaC abreraKaren.pdf

Del Castillo Palacios, R. A. (2018). Diseño e implementación del sistema de gestión ambiental basado en la ISO 14001:2015 para la estación de servicios grifo Chavín. Huari. 2017. Huaraz, Ancash, Perú: Universidad Nacional "Santiago Antunez de Mayolo".

http://repositorio.unasam.edu. pe/bitstream/handle/UNASA M/2182/T033_40826462_T.p df?sequenc

Enríquez Bernal, L. M. (2009). Estrategia para la implementación de la norma ISO 14001en empresas productoras de palma de aceite de la zona Oriental Colombiana. Colombia: Pontificia Universidad Javeriana.

https://repository.javeriana.ed u.co/bitstream/handle/10554/ 717/eam36. pdf?sequence $=1$ \&isAllowed $=\mathrm{y}$

Escuela Europea de Excelencia. (2015). ISO 14001: ¿Cómo implementar un Sistema de Gestión Ambiental? https://www.nueva-iso14001.com/2014/12/iso14001-revision-ambientalinicial/

Espín Guerrero, R. D. (2018). Sistema de gestión ambiental basado en la norma ISO 14001-2015 para la empresa
"Maquinarias Espín". Ambato - Ecuador: Universidad Técnica de Ambato. https://repositorio.uta.edu.ec/ bitstream/123456789/27597/ 1/Tesis_t1379id.pdf

Guzmán Ramos, A. (2001). La problemática ambiental desde la perspectiva geográfica. Barcelona: REVISTA BIBLIOGRÁFICA DE GEOGRAFÍA Y CIENCIAS SOCIALES.

http://www.ub.edu/geocrit/b3 w-296.htm

Huatuco Mamani, S. Y. (2019). Determinación de aspectos ambientales asociados a las actividades del centro de salud ocupacional San Miguel para la implementación de un sistema de gestión ambiental ISO 14001:2015. Puno - Perú: Universia Nacional del Altiplano. http://repositorio.unap.edu.pe /bitstream/handle/UNAP/1070 6/Huatuco_Mamani_Siomara _Yelka.pdf?sequence $=1$ \&isAl lowed=y

Lara Lascano, C. J. (2014). Desarrollo de la metodología para la implementación de un sistema de gestión ambiental conforme la norma ISO 14001:2004 para Ecuamatriz CÍA. LTDA. Ambato. Riobamba - Ecuador: Escuela Superior Politécnica de Chimborazo. http://dspace.espoch.edu.ec/ 
bitstream/123456789/3387/1/

85T00296.pdf

Norma ISO 14001. (2015). Sistema de gestión ambiental requisitos interpretación norma ISO 14001:2015.

Presidencia de la Republica. (2017).

Código Orgánico del

Ambiente.

https://www.ambiente.gob.ec/

wp-

content/uploads/downloads/2

018/09/Codigo-Organico-del-

Ambiente.pdf

Rojas González, M. (2010). ISO 14001.

http://ingenieria83.blogspot.c om/2010/12/iso-14001.html

Salazar Terán, A. G. (2017). Diseño del sistema de gestión ambienta basado en la norma ISO 14001:2015 para la empresa Servicios Mecánicos "SMO". Quito: Universidad Central del Ecuador. http://www.dspace.uce.edu.e c/bitstream/25000/11531/1/TUCE-0017-0025-2017.pdf 